OPEN ACCESS

Edited by:

Pietro Pietrini,

IMT School for Advanced Studies

Lucca, Italy

Reviewed by:

Sushil K. Jha,

Jawaharlal Nehru University, India

Stefano Landi,

University of Pisa, Italy

*Correspondence:

Lyn R. Griffiths

lyn.griffiths@qut.edu.au

Received: 22 December 2016 Accepted: 25 April 2017 Published: 16 May 2017

Citation:

Avgan N, Sutherland HG, Lea RA, Spriggens $L K$, Haupt $L M$, Shum DHK and Griffiths LR (2017) A CREB1 Gene Polymorphism (rs2253206) Is Associated with

Prospective Memory in a Healthy

Cohort.

Front. Behav. Neurosci. 11:86. doi: 10.3389/fnbeh.2017.00086

\section{A CREB1 Gene Polymorphism (rs2253206) Is Associated with Prospective Memory in a Healthy Cohort}

\author{
Nesli Avgan', Heidi G. Sutherland ${ }^{1}$, Rodney A. Lea ${ }^{1}$, Lauren K. Spriggens' ${ }^{2}$, \\ Larisa M. Haupt ${ }^{1}$, David H. K. Shum ${ }^{2}$ and Lyn R. Griffiths ${ }^{1 *}$
}

${ }^{1}$ Genomics Research Centre, Chronic Disease and Ageing, Institute of Health and Biomedical Innovation, School of Biomedical Sciences, Queensland University of Technology, Brisbane, QLD, Australia, ${ }^{2}$ Menzies Health Institute Queensland and School of Applied Psychology, Griffith University, Gold Coast, QLD, Australia

Prospective memory (PM) is generally defined as remembering to perform intended actions in the future and is important for functioning in daily life. Cyclic adenosine monophosphate (CAMP) responsive element binding protein 1 (CREB1) plays an important role in cognitive functions. In this study, we hypothesized that genetic variation in the CREB1 gene is associated with PM. We genotyped a CREB1 promoter polymorphism rs2253206 and tested it for association with PM in 619 healthy subjects. PM performance was measured using the Prospective and Retrospective Memory Questionnaire (PRMQ), the Comprehensive Assessment of Prospective Memory (CAPM), and the Memory for Intentions Screening Test (MIST). Generalized linear model analysis was conducted for each PM test independently using different inheritance models to identify any associations $(p<0.05)$. After multiple testing adjustments, a significant association was found between the rs2253206 genotype and PM performance for CAPM instrumental activities of daily living measure $(p=0.016)$. These results suggest that the rs2253206 polymorphism in the CREB1 gene locus is associated with PM in healthy individuals and contributes to knowledge on the genetics of this particular type of memory.

Keywords: CREB1, prospective memory, genetics of human memory, polymorphism, rs2253206

\section{INTRODUCTION}

Memory is a complex neurocognitive function, important in humans to establish an understanding of the past, present, and future (Kandel, 2006). In Squire's widely accepted neuropsychological model of human memory, memory is divided into two subgroups: declarative and non-declarative memory. Declarative memory represents our conscious recall and is further divided into two subtypes, semantic and episodic memory (Squire, 1992). Episodic memory stores autobiographic events and semantic memory stores facts, concepts, and our knowledge (Tulving, 1985; Kandel, 2013). Collectively, these defined memory types refer to the past, and recollection of pieces of information from the past, further generalized under the term retrospective memory.

For remembering our intentions after a delay, we use prospective memory (PM). PM is essential for our understanding and sense of the future and refers to the function that enables a person to carry out a planned act after a delay (Burgess et al., 2001). Remembering to remember is the 
basic definition of PM and it can be divided into time- and eventbased sub-types, and can also be triggered by cues (Baddeley et al., 2009; Burgess et al., 2011; Raskin et al., 2012). Remembering to call a friend at a certain time is an example of a time-based PM. Whereas if a friend asks you to give them a call when dinner is ready, remembering and calling the friend is an example of an event-based PM. Forgetting to call your friend at that required time, but remembering that you need to call them after seeing someone on the phone is an example for how PM can be triggered by a cue. One of the age-related memory impairments is PM failure, or forgetting intentions (Einstein et al., 2000). Dementia, Parkinson's disease, Alzheimer's disease, and schizophrenia also show PM impairments (Huppert and Beardsall, 1993; Jones et al., 2006; Woods et al., 2007; Kliegel et al., 2011). To date, little is known of the genetic factors which contribute to PM and its impairment, but as genetic variants have been shown to be associated with performance in a variety of memory subtypes, they are likely to also contribute to PM.

The cyclic adenosine monophosphate (cAMP) pathway has been shown to play an essential role in memory and cognitive abilities (Kandel, 2012). The cAMP responsive element binding protein 1 (CREB1) gene encodes a transcription factor that is a member of the leucine zipper family of DNA binding proteins (Quinn and Granner, 1990; Taylor et al., 1990). Studies have demonstrated the importance of CREB family proteins in learning and memory (Mizuno et al., 2002). CREB1 is a member of a family of proteins that function as transcription factors expressed in the brain (Kandel, 2012) with CREB signaling central to spatial, associative, emotional, and social memories in mammals (Gass et al., 1998; Pittenger et al., 2002; Viosca et al., 2009). Studies in mice and rats have described the role of CREB as a universal modulator of memory formation (Lazary et al., 2011). In humans, cognitive disorders and neurodegeneration disease studies on Huntington's disease, Alzheimer's disease, Rubinstein-Taybi syndrome, and CoffinLowry syndrome, support a key role for CREB signaling (Sweatt, 2009). Furthermore, CREB has been shown to play an essential role in synaptic plasticity, a feature of the pathways of memory formation (Kanehisa, 2002; Mantamadiotis et al., 2002; Saura and Valero, 2011).

Several studies focusing on depression, schizophrenia, rumination, and negative emotionally related memory impairments have found significant associations with CREB regulation (Kawanishi et al., 1999; Barrot et al., 2002; Lazary et al., 2011; Guo et al., 2014). Genetic polymorphisms can influence the expression of linked loci. Lazary et al. (2011) reported the involvement of rumination in memory and identified significant associations between the GG genotype of rs2253206 in CREB1 and rumination and negative emotionality in two independent Caucasian cohorts $(n=651$ and $n=1174)$. The rs2253206 polymorphism is located in the promoter region of CREB1 gene (Sands and Palmer, 2008; Rankinen et al., 2010). Furthermore, Rankinen et al. (2010) revealed that the CREB1 polymorphism rs2253206 major $\mathrm{G}$ allele is associated with significantly lower promoter activity than the minor A allele. Given these results we hypothesized that, as CREB1 plays an important role in cognition and memory, polymorphisms that influence CREB1 expression may impact on memory performances of individuals.

In the present work, we conducted an association study focusing on the rs2253206 polymorphism in CREB1 and PM performance. We investigated six different aspects of PM examined by three different memory tests in a quantitative manner, and report significant associations for some of these test scores with the CREB1 rs2253206 polymorphism in a healthy cohort of individuals.

\section{MATERIALS AND METHODS}

\section{Study Participants}

Individuals $(n=619)$ were recruited through advertisements at the Queensland University of Technology (QUT) and Griffith University campuses, with volunteers also invited to participate by posters and advertisements displayed around local shopping centers and health clinics for memory testing and subsequent genotyping. Participation was excluded for individuals with a history of psychiatric disorder or head injury to maintain a representative sample of cognitive and memory ability without additional complications. Due to the participation criteria, six individuals with a history of psychological background were excluded from the study and 12 additional participants were excluded after genotyping to maintain a stringent confidence interval (99\%) in the data set. Thus, the study examined 601 healthy individuals (429 females and 172 males; median age: 20; range; 16-65 years.). Participants largely reported English to be their first language (89\%), identified as mostly Australian (74\%) in ethnicity and had varying education levels. All 601 participants met the study requirements of non-pathological healthy individuals. The study was approved by the Griffith University (MSC/01/09/HREC) and QUT Human Research and Ethics (1300000486) Committees. Written informed consent was provided by all participants prior to any study activities. Participants undertook a comprehensive battery of memory tests and completed self-report questionnaires to gauge their memory status. Two subsets (vocabulary and matrix reasoning) of The Wechsler Abbreviated Scale of Intelligence (WASI) were completed to estimate the intelligence quotient (IQ) of participants.

\section{Memory Testing for PM}

We have employed three memory battery tests comprised of six memory subtests to assess memory performances of the participants, and utilized each score as independent phenotypes, since each measurement has different combination of elements. A relevant subset of questions $(n=8)$ in the Prospective and Retrospective Memory Questionnaire (PRMQ) was used to measure PM. PRMQ evaluates memory failures on a 5-point scale ranging from never to very often, concerning everyday life basis memory tasks and this questionnaire creates a three-dimensional result by combining long-term and short-term memory, and cue factors while assessing PM. An example for this test can be given as "Do you forget to buy something you planned to buy, like a birthday card, even when you see the shop?" (Smith et al., 2000; 
Crawford et al., 2003). For measuring PM capacity in addition to PRMQ, the Comprehensive Assessment of Prospective Memory (CAPM) test was also undertaken. This questionnaire has the same ranging scale as the PRMQ. It defines the frequency of PM failures in everyday life in regard to instrumental activities of daily living (IADL) and basic activities of daily living (BADL). Examples for IADL and BADL are "Leaving the iron on" or "Not locking the door when leaving home," respectively (Chau et al., 2007). For the above tests, a higher score reflects more memory failures and therefore poorer PM. Furthermore, Memory for Intentions Screening Test (MIST) trials were administered to the participants to measure the execution of PM, including time and event based, with and without cues. The MIST has two sub-scores, the immediate MIST score (MIST-IMD) comprised of eight PM tasks which participants perform during the memory evaluation such as "When I show you a red pen, sign your name on the paper," as well as a delay score (MIST-Delay), in which they were asked to email the examiner the next day at a certain time to address a query by the examiner (Woods et al., 2008; Kamat et al., 2014). For MIST, a higher score reflects better PM performance.

\section{Genotyping}

Saliva samples were collected from each participant immediately after completion of the memory tests using Oragene ${ }^{\circledR}$ DNA Self-Collection kits (DNA Genotek Inc., Ottawa, ON, Canada). DNA was extracted from whole saliva samples using the kit and protocol of the same manufacturer. The rs2253206 polymorphism in the CREB1 gene was genotyped using the MassARRAY $^{\circledR}$ System by Agena Bioscience ${ }^{\text {TM }}$ (San Diego, CA, USA) on a 96-well MassARRAY platform. Amplification and extension primers used to detect the variant were designed with the Assay Design Suite (Agena Bioscience ${ }^{\mathrm{TM}}$ ) and purchased from IDT (Singapore). The assay is based on the mass of the post-polymerase chain reaction (PCR) single base extension as previously described (Gabriel et al., 2009).

\section{Data Analysis}

All descriptive statistics of the population and the phenotype data were carried out using The R Program for Statistical Computing (v3.2.2; R Development Core Team, 2015). Correlations between selected memory tests (PRMQ-PM, CAPM-IADL, CAPM-BADL, CAPM-Total, MIST-IMD, MIST-Delay) and IQ were investigated using Pearson's $r$ test. Quality control (QC) of the data and quantitative genetic analyses were conducted using PLINK (v1.09; Purcell et al., 2007). Due to the constructional differences of each assessment, we chose to treat memory scores individually as independent hypotheses and therefore did not adjust for multiple testing. Generalized linear model analysis for each memory phenotype individually was conducted using an additive model with a statistical significance level of $p<0.05$ used to identify associations. Age, sex, and IQ were considered as covariates in the analysis. Analyses were also performed for dominant and recessive models of inheritance to compare the beta scores of all models to estimate genotypic effects on memory status. Consequently, to avoid false positive results we have accounted for multiple testing with respect to using three inheritance models and adjusted our $p$-value threshold using Bonferroni correction by setting an alpha-level of 0.0166 as the statistical significance threshold. Finally, we have further investigated the CREB1 gene and the rs2253206 polymorphism using Genotype-Tissue Expression (GTEx) Browser ${ }^{1}$ to study the differences in tissue-specific levels by expression quantitative trait loci (eQTL) analysis (GTEx Consortium, 2013), and LDlink to explore the linkage disequilibrium (LD) between rs2253206 and the single nucleotide polymorphisms (SNPs) in close proxy, using all 1000 Genomes populations, to find putatively functional variants in our region of interest (Machiela and Chanock, 2015).

\section{RESULTS}

In this study, PM performance was measured using three memory tests widely used to assess memory impairments (Raskin et al., 2012): PRMQ, CAPM, and MIST. PRMQ and CAPM are self-report questionnaires. PRMQ evaluates RM and PM with equal number of questions, but in this study only the aspect that assesses PM was used, which focuses on common everydaylife memory failures such as forgetting to take a pill (Smith et al., 2000). CAPM assesses two different PM failures; basic daily routine failures such as forgetting to eat a meal or forgetting to put on a piece of clothing (e.g., socks) are measured using BADL, and instrumental activity related failures such as leaving the stove on or having trouble remembering personal dates at the right time (e.g., birthdays) are measured using IADL (Chau et al., 2007). The MIST is a one-on-one task-based test measuring time- and event-based planned acts with and without cues, using eight different PM tasks while solving a word-search puzzle as a distraction (Kamat et al., 2014). Examples of MIST for PM assessment include: "In exactly 15 min please tell me it is time to take a break" for time based; and "When I hand you a red pen, please sign your name on your paper" for event based with a cue. MIST also includes a delay test in which participants were asked to send an email to the examiner at a certain time, answering a specific question asked during the test (Woods et al., 2008; Kamat et al., 2014). We have studied these memory measurements independently due to the distinct elements of each assessment.

\section{Descriptive Statistics of the Memory Cohort}

As a result of the SNP array QC step, 12 individuals were excluded from further analyses due to poor genotyping quality. Genetic analysis was performed on 601 healthy individuals with IQ normally distributed in the samples, ranging from 78 to 138 with a mean IQ of 108 (SD = 10.77). Demographics of the memory cohort are presented in Table 1. Two-thirds of the cohort was female $(71 \%)$. The age of participants ranged from 16 to 65 years and the majority of the cohort were Australian. With respect to education determined by the highest qualification, the majority of the individuals in the cohort were high school graduates, but a large number were continuing to a diploma or a bachelor's degree due to the high number of individuals in the younger age class (16-25 years) present in the cohort.

\footnotetext{
${ }^{1}$ http://www.gtexportal.org/home/
} 
TABLE 1 | Demographics of the Genomics Research Centre (GRC) memory cohort.

\begin{tabular}{lc}
\hline Variable & Participants $(\boldsymbol{n}=\mathbf{6 0 1}), \boldsymbol{N}(\mathbf{\%})$ \\
\hline Age group & $469(77.04)$ \\
$16-25$ & $79(13.14)$ \\
$26-35$ & $36(6.00)$ \\
$36-45$ & $10(1.66)$ \\
$46-55$ & $7(1.16)$ \\
$56-65$ & \\
Gender & $172(28.62)$ \\
Male & $429(71.38)$ \\
Female & \\
Ethnicity & $446(74.88)$ \\
Australian & $155(25.12)$ \\
Other & \\
Highest qualification & $376(62.56)$ \\
School certificate & $143(23.79)$ \\
Diploma program & $59(9.82)$ \\
Bachelor's degree & $21(3.49)$ \\
Postgraduate degree & $2(0.33)$ \\
Other &
\end{tabular}

Correlation analysis showed that IQ was not correlated with any of the memory tests. When we look further into subtests of CAPM, CAPM-Total was strongly correlated within CAPM-IADL $(r=0.83)$ and CAPM-BADL $(r=0.79)$, which is expected because the CAPM-Total is made up of these two sub-scores. Additionally, the PRMQ and CAPM-Total were moderately correlated with each other $(r=0.58)$. This may reflect the nature of both tests; both are self-answered questionnaires focused on daily memory failures, yet they do have some constructive differences. In contrast, despite the use of MIST as a PM measure we did not find it to be correlated with either PRMQ or CAPM. Of note, MIST is not a questionnaire but a memory test, administered by the examiner and requires behavioral responses.

\section{Genetic Association Analysis}

The rs2253206 SNP passed QC and genotype distribution was found to be in Hardy-Weinberg equilibrium (>0.05). Genetic association analysis was then completed using age, gender, and IQ as covariates, to focus solely on the effect of the polymorphism on PM performance. Recessive, additive, and dominant models of inheritance were undertaken with linear regression analysis to consider possible allelic affects and to account for multiple testing for these models we have adjusted the significance threshold for this study by using Bonferroni correction, setting the significance $p$-value to 0.0167 . Results are summarized in Table 2. A significant association was identified between the rs2253206 variant and the CAPM-IADL memory phenotype in the additive model [ $\left.p_{\mathrm{CAPM}(\mathrm{IADL})}=0.016\right]$. Additionally, we found nominal associations for CAPM-IADL in the recessive model $\left[p_{\text {CAPM }(I A D L)}=0.033\right]$ and the MIST-IMD memory phenotype in the dominant model [ $p_{\mathrm{MIST}(\mathrm{IMD})}=0.039$ ]

The frequency of the minor A-allele for the rs2253206 polymorphism in our cohort was calculated to be 0.437 , with genotype distributions of $19.1 \%$ for AA, $49.6 \%$ for AG, and $31.3 \%$ for GG genotypes (Table 3 ). Individuals carrying the A-allele showed a stronger association with the CAPM-IADL test results in the additive model of inheritance compared with the recessive model of inheritance ( $p=0.016$ vs. $p=0.033$ ). In the CAPM test, a higher score reflects poorer memory performance and Table 3 shows that the A-allele is associated with lower CAPM-IADL memory test scores, corresponding to less memory failures and a better PM performance. In the MIST, a higher score reflects better memory performance and Table 3 shows that for MIST-IMD the A-allele was correlated with higher test scores. Therefore, the significant results of CAPM-IADL (additive model) and nominally significant results of MIST-IMD (dominant model) in the same population are consistent with the minor A-allele of rs2253206 being correlated with better PM performance. Furthermore, the direction of the beta score obtained for the MIST-IMD association analysis was opposite that obtained for the CAPM-IADL test as would be expected by this suggestion (Table 2). In the PRMQ-PM test higher test scores reflect poorer

TABLE 2 | CREB1 variant rs2253206 association with memory phenotypes for recessive, additive and dominant model of inheritance; controlling for IQ, gender, and age.

\begin{tabular}{|c|c|c|c|c|c|c|c|c|c|c|}
\hline \multirow[t]{2}{*}{ Memory test } & \multirow[t]{2}{*}{ Assessed memory type } & \multicolumn{3}{|c|}{ Recessive model } & \multicolumn{3}{|c|}{ Additive model } & \multicolumn{3}{|c|}{ Dominant model } \\
\hline & & $\beta$ & $t$ & $p$ & $\beta$ & $t$ & $p$ & $\beta$ & $t$ & $p$ \\
\hline \multirow[t]{4}{*}{ CAPM } & Prospective memory & & & & & & & & & \\
\hline & IADL & -0.124 & -2.141 & 0.033 & -0.079 & -2.424 & 0.016 & -0.091 & -1.838 & 0.067 \\
\hline & BADL & -0.039 & -0.840 & 0.401 & -0.033 & -1.260 & 0.208 & -0.046 & -1.190 & 0.235 \\
\hline & Total & -0.052 & -1.161 & 0.246 & -0.041 & -1.624 & 0.105 & -0.056 & -1.466 & 0.143 \\
\hline \multirow[t]{3}{*}{ MIST } & Prospective memory & & & & & & & & & \\
\hline & Immediate & 0.059 & 0.371 & 0.711 & 0.141 & 1.576 & 0.116 & 0.280 & 2.068 & 0.039 \\
\hline & Delay & 1.164 & 1.238 & 0.216 & 0.861 & 1.630 & 0.104 & 1.125 & 1.409 & 0.159 \\
\hline PRMQ & Prospective memory & -0.624 & -1.309 & 0.191 & -0.425 & -1.588 & 0.113 & -0.520 & 1.285 & 0.199 \\
\hline
\end{tabular}

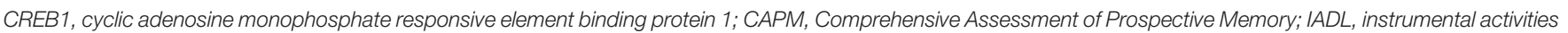

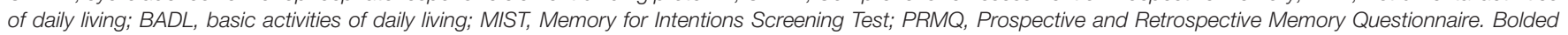
values shows the $p<0.05$. 
TABLE 3 | CREB1 variant rs2253206 allelic distribution and allele counts, with memory and IQ score distributions.

\begin{tabular}{|c|c|c|c|c|}
\hline \multirow[t]{2}{*}{ Test } & \multirow[t]{2}{*}{ Assessed memory type } & \multirow{2}{*}{$\begin{array}{c}\text { AA }(n=115) \\
\text { Mean (min-max) }\end{array}$} & \multirow{2}{*}{$\begin{array}{c}\text { AG }(n=297) \\
\text { Mean (min-max) }\end{array}$} & \multirow{2}{*}{$\begin{array}{c}\text { GG }(n=189) \\
\text { Mean (min-max) }\end{array}$} \\
\hline & & & & \\
\hline \multirow[t]{4}{*}{ CAPM } & Prospective memory & & & \\
\hline & IADL & $2.048(1.00-3.88)$ & $2.143(1.00-4.43)$ & $2.224(1.13-4.00)$ \\
\hline & BADL & $1.515(1.00-3.25)$ & $1.540(1.00-3.25)$ & $1.581(1.00-3.13)$ \\
\hline & Total & $1.796(1.062-3.13)$ & $1.828(1.00-3.27)$ & $1.885(1.13-3.5)$ \\
\hline \multirow[t]{3}{*}{ MIST } & Prospective memory & & & \\
\hline & Immediate & $14.700(5.00-16.00)$ & $14.740(9.00-16.00)$ & $14.440(6.00-16.00)$ \\
\hline & Delay & $1.365(0.00-2.00)$ & 1.279 (0.00-2.00) & $1.185(0.00-2.00)$ \\
\hline$P R M Q$ & Prospective memory & $20.380(10.00-38.00)$ & $20.820(12.00-38.00)$ & $21.330(11.00-37.00)$ \\
\hline IQ & & $108.522(80-130)$ & $108.250(78-138)$ & 107.598 (78-134) \\
\hline
\end{tabular}

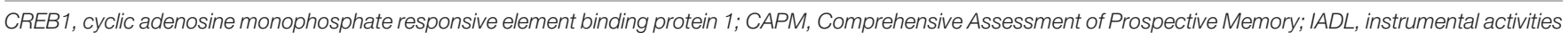

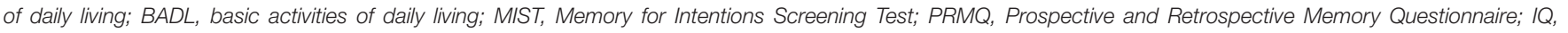
Intelligence Quotient.

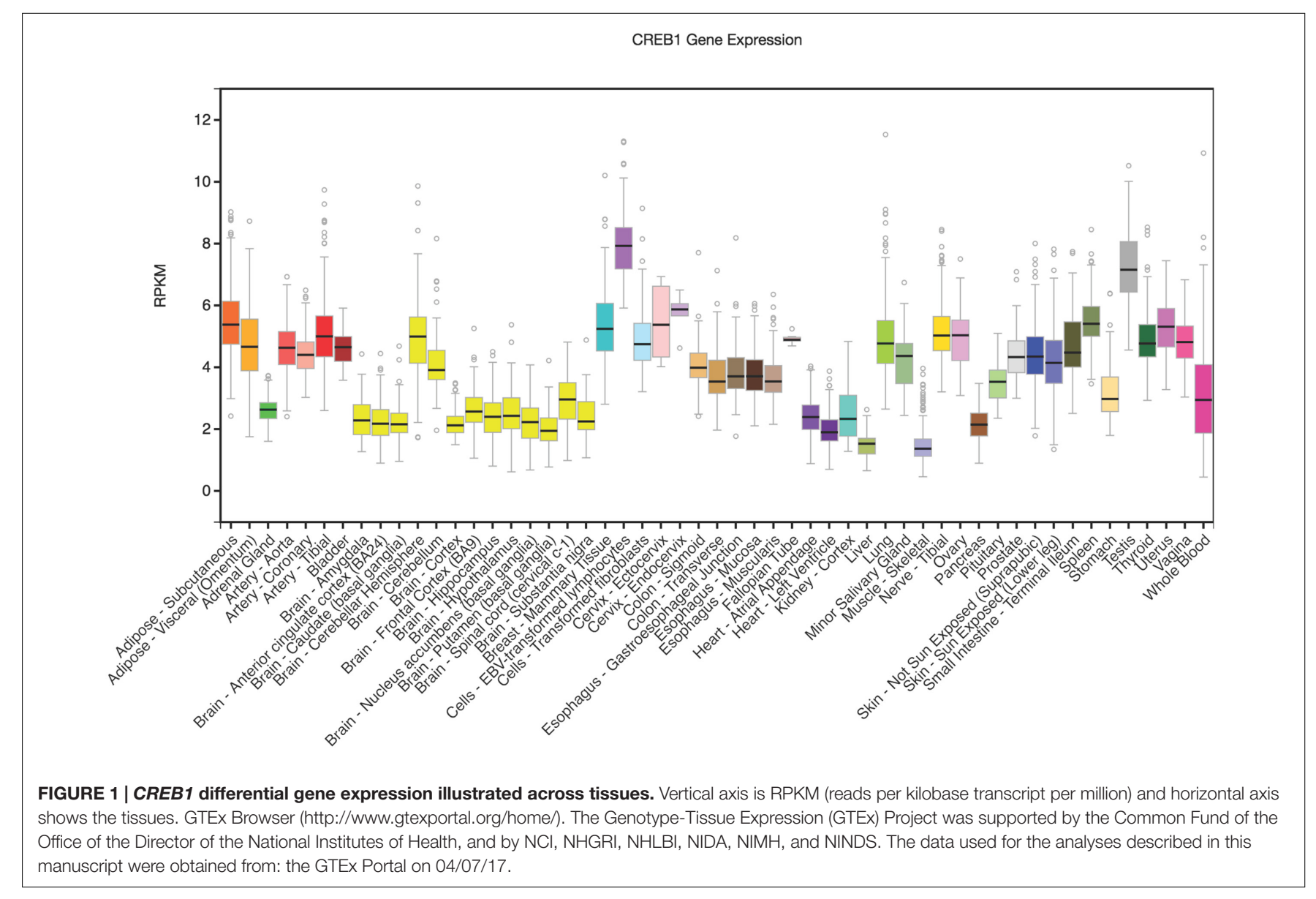

memory performance. While the results for PRMQ-PM were not significant, the A-allele was also correlated with better PM performance, as well as higher IQ scores (Table 3).

Rankinen et al. (2010) reported that the minor A-allele of rs2253206 was associated with lower CREB1 promoter activity compared to the G-allele in transfected mouse skeletal muscle cell lines. In order to further explore how rs2253206 might affect CREB1 expression in areas of the brain that are involved in memory processes we used the GTEx eQTL Browser to investigate CREB1 mRNA levels. CREB1 is expressed in brainrelated tissues (shown in yellow) and most highly in the cerebellum, but is at lower levels than some tissues where it is highly expressed such as testis (shown in gray) and transformed lymphocytes (shown in purple) (Figure 1).

We then queried GTEx for evidence of eQTLs of rs2253206. CREB1 was not reported as an eQTL of the rs2253206 


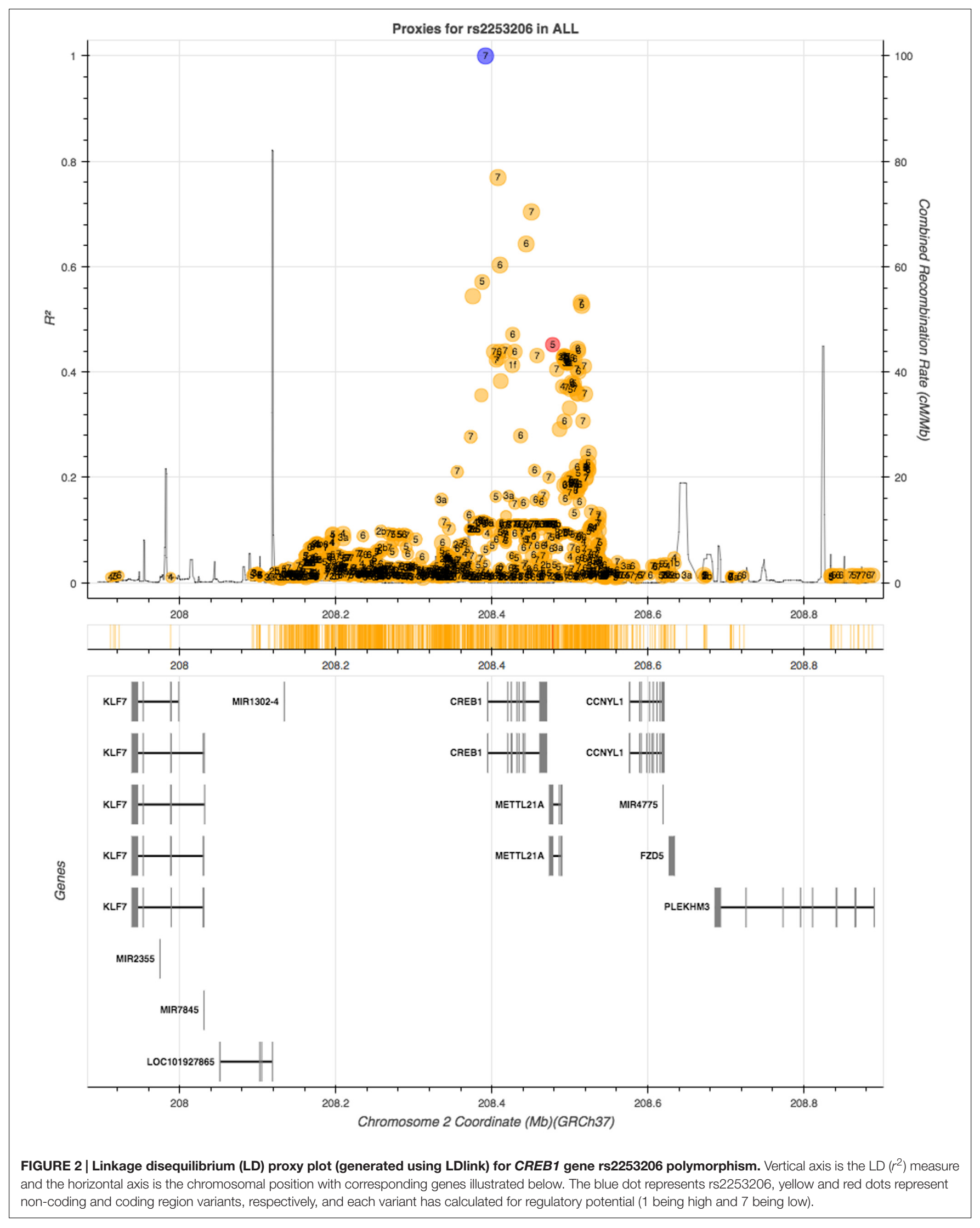


polymorphism in GTEx, however, a gene that overlaps with CREB1, methyltransferase like 21A (METTL21A), and a downstream gene, cyclin Y-like 1 (CCNYL1), are eQTLs for rs2253206 in some non-neural tissues. To determine whether any SNPs in high LD with rs2253206 may be influencing either CREB1 expression levels or its protein we looked for SNPs that are in close proximity to rs2253206 polymorphism using LDlink, an online tool to investigate LD in population groups for a polymorphism or loci (Machiela and Chanock, 2015). Using 1000 Genomes data (phase 3) with all the populations, we could not identify any other polymorphism or functional variants in high LD $\left(r^{2}>0.8\right)$ with rs2253206 that could have provided further information to support explaining the effect of the rs2253206 polymorphism (Figure 2).

\section{DISCUSSION}

In this study, we used a non-pathological population-based sample of individuals to investigate the effect of the rs2253206 CREB1 gene polymorphism on PM. After multiple testing corrections, we found that the minor A-allele at rs2253206 was significantly associated with better PM performance in an additive model of inheritance and the homozygous AA genotype was nominally associated in a recessive model of inheritance. In contrast, the homozygous major $G$ allele was shown to be correlated with poorer PM and IQ scores (Tables 2, 3). The difference between the memory test scores for the three genotypes (AA, AG, and GG) are small and this is likely to due to the fact that the cohort is comprised of healthy, and mainly young, individuals. Nevertheless, small increments in the scores obtained in the PRMQ, CAPM, and MIST can reflect a large impact on memory performance and daily living. While these tests are commonly used to assess elderly individuals or those that might be affected by Alzheimer's disease, Parkinson's disease, schizophrenia, etc., our results suggest that genetic factors may contribute to performance of PM tasks in healthy individuals.

Two of the memory measures used in this study, PRMQ-PM and CAPM-BADL, are very similar in the questions asked, yet they are not highly correlated due to the different construction of the tests. Neither test was found to be significantly associated with rs2253206 in the study cohort. MIST-IMD, which evaluates PM behavioral performance with respect to immediate memory, was nominally associated with rs2253206, while MIST-Delay which measures another aspect of PM encompassing long-term memory, did not show any association. We also found that MISTIMD and MIST-Delay test scores were not correlated with each other. All tests assess measures of PM, but lack of correlation between test scores suggest that it is a heterogeneous construct.

Several studies have demonstrated that CREB is essential for long-term memory and synaptic plasticity (Impey et al., 1996; Lamprecht et al., 1997; Josselyn and Nguyen, 2005; Alberini, 2009; Wallace et al., 2009). Additionally, Williams et al. (2008) have shown changing expression levels of CREB influences working memory in a rat model. SNPs in the CREB1 gene, including rs2253206, have previously been significantly associated with traits linked to cognitive vulnerability. Guo et al.
(2014) investigated the effect of CREB1 gene polymorphisms on cognitive dysfunction in Chinese patients with major depression and their results suggest the CREB1 gene is a promising marker for cognitive function in major depression patients. Juhasz et al. (2011) investigated risk factors for depression, and while studying the neuroplastic pathway, they demonstrated a significant association between the CREB1 rs2253206 polymorphism with rumination and severe depression, reporting that minor allele carriers are less likely to ruminate. These results suggest that CREB1 rs2253206 is, or is linked with, a functional SNP. However, we were unable to find evidence that CREB1 is an eQTL of rs2253206 using the GTEx database, although it suggested that the nearby genes METTL21A and CCNYL1 were in some nonneuronal tissues. Little is known of the function of these genes. Furthermore, we could not detect any other polymorphisms in high LD with rs2253206 that suggested a role in CREB1 expression or function.

Functional analysis of the rs2253206 SNP by Rankinen et al. (2010) demonstrated that the major G-allele has lower promoter activity, likely due to loss of a CCAAT enhancer-binding protein alpha $(\mathrm{C} / \mathrm{EBP} \alpha)$ transcription factor binding site. Therefore, the effect of rs2253206 on cognitive function observed in our cohort may occur via altered expression levels of CREB1. While analysis of CREB1 expression using GTEx did not show high levels in brain-related tissues, $C R E B 1$ transcripts were present in all neural tissues and highest in cerebellar tissues. Although that part of the brain has not been reported to be particularly associated with PM, Tomlinson et al. (2014) found contribution of the cerebellum in working memory.

A limitation for our study is the small sample size which impacts on the power to find significant associations. Nevertheless, our findings suggest further investigation of the CREB pathway in our memory cohort may be justified, and also that the rs2253206 polymorphism should be tested in a similarly characterized independent cohort. However, with respect to PM few studies have investigated the genetic factors involved and thus have both PM phenotypes and genotyping data available. We have previously reported a significant effect of the APOE $\varepsilon 4$ polymorphism in PM using the CAPM test in a smaller subset of the cohort investigated here (Donges et al., 2012). Evans et al. (2014) also reported an association of APOE $\varepsilon 4$ genotype with PM using different PM tasks to our study; interestingly they found that genotype affects changed with age of the participants (Evans et al., 2014). Kennedy et al. (2015) investigating the brain-derived neurotrophic factor (BDNF) Val66Met polymorphism (rs6265) also found an adverse effect of age on PM performance across the lifespan which was much stronger in the BDNF Met carriers than for the Val homozygotes. Therefore, it would be of interest to also study the CREB1 rs2253206 polymorphism in an older cohort tested for PM performance, when impairments influenced by genetic factors may become more pronounced.

In summary, we have identified associations between CREB1 rs2253206 and PM in a healthy cohort; one of the memory phenotypes (CAPM-IADL) was identified as being significantly associated and another (MIST-IMD) identified as nominally associated with rs2253206. When we consider its location and evidence for an effect of the rs2253206 polymorphism on CREB1 
promoter activity, it is conceivable that genotypic variation may affect $\mathrm{PM}$ performance via a regulatory effect on the CREB1 gene, a gene known to be involved in cognition and re-modeling of synaptic plasticity. Our results suggest that further studies of polymorphisms in CREB1 and other genes in the CAMP pathway would be of interest to explore its role in the genetic basis of PM.

\section{AUTHOR CONTRIBUTIONS}

NA performed the laboratory work, data analysis, and interpretation. HS and RL supervised the study and interpretation of the results. DS designed the memory

\section{REFERENCES}

Alberini, C. M. (2009). Transcription factors in long-term memory and synaptic plasticity. Physiol. Rev. 89, 121-145. doi: 10.1152/physrev.00017.2008

Baddeley, A. D., Eysenck, M. W., and Anderson, M. (2009). Memory. Hove: Psychology Press.

Barrot, M., Olivier, J. D., Perrotti, L. I., DiLeone, R. J., Berton, O., Eisch, A. J., et al. (2002). CREB activity in the nucleus accumbens shell controls gating of behavioral responses to emotional stimuli. Proc. Natl. Acad. Sci. U.S.A. 99, 11435-11440. doi: 10.1073/pnas.172091899

Burgess, P. W., Gonen-Yaacovi, G., and Volle, E. (2011). Functional neuroimaging studies of prospective memory: what have we learnt so far? Neuropsychologia 49, 2246-2257. doi: 10.1016/j.neuropsychologia.2011.02.014

Burgess, P. W., Quayle, A., and Frith, C. D. (2001). Brain regions involved in prospective memory as determined by positron emission tomography. Neuropsychologia 39, 545-555. doi: 10.1016/S0028-3932(00)00149-4

Chau, L. T., Lee, J. B., Fleming, J., Roche, N., and Shum, D. (2007). Reliability and normative data for the comprehensive assessment of prospective memory (CAPM). Neuropsychol. Rehabil. 17, 707-722. doi: 10.1080/09602010600923926

Crawford, J. R., Smith, G., Maylor, E. A., Della Sala, S., and Logie, R. H. (2003). The Prospective and Retrospective Memory Questionnaire (PRMQ): normative data and latent structure in a large non-clinical sample. Memory 11, 261-275. doi: 10.1080/09658210244000027

Donges, B., Haupt, L. M., Lea, R. A., Chan, R. C., Shum, D. H., and Griffiths, L. R. (2012). Role of the apolipoprotein E and catechol-O-methyltransferase genes in prospective and retrospective memory traits. Gene 506, 135-140. doi: 10.1016/j.gene.2012.06.067

Einstein, G. O., McDaniel, M. A., Manzi, M., Cochran, B., and Baker, M. (2000). Prospective memory and aging: forgetting intentions over short delays. Psychol. Aging 15, 671-683. doi: 10.1037/0882-7974.15.4.671

Evans, S., Dowell, N. G., Tabet, N., Tofts, P. S., King, S. L., and Rusted, J. M. (2014). Cognitive and neural signatures of the APOE E4 allele in mid-aged adults. Neurobiol. Aging 35, 1615-1623. doi: 10.1016/j.neurobiolaging.2014.01.145

Gabriel, S., Ziaugra, L., and Tabbaa, D. (2009). SNP genotyping using the Sequenom MassARRAY iPLEX platform. Curr. Protoc. Hum. Genet. Chapter 2, Unit2.12. doi: 10.1002/0471142905.hg0212s60

Gass, P., Wolfer, D. P., Balschun, D., Rudolph, D., Frey, U., Lipp, H. P., et al. (1998). Deficits in memory tasks of mice with CREB mutations depend on gene dosage. Learn. Mem. 5, 274-288.

Guo, J., Liu, Z., Dai, H., Zhu, Z., Wang, H., Yang, C., et al. (2014). Preliminary investigation of the influence of CREB1 gene polymorphisms on cognitive dysfunction in Chinese patients with major depression. Int. J. Neurosci. 124, 22-29. doi: 10.3109/00207454.2013.816956

GTEx Consortium (2013). The genotype-tissue expression (GTEx) project. Nat. Genet. 45, 580-585. doi: 10.1038/ng.2653

Huppert, F. A., and Beardsall, L. (1993). Prospective memory impairment as an early indicator of dementia. J. Clin. Exp. Neuropsychol. 15, 805-821. doi: 10.1080/01688639308402597

Impey, S., Mark, M., Villacres, E. C., Poser, S., Chavkin, C., and Storm, D. R. (1996). Induction of CRE-mediated gene expression by stimuli that generate performance evaluations. LS participated in phenotype data collection. LH and LG supervised the study. All authors contributed to writing and editing of the manuscript.

\section{FUNDING}

This work was supported by an Australian Research Council grant The Genetic Basis of Human Memory (Project ID: DP130101921). This work was also supported by infrastructure purchased with Australian Government EIF Super Science Funds as part of the Therapeutic Innovation Australia - Queensland Node project.

long-lasting LTP in area CA1 of the hippocampus. Neuron 16, 973-982. doi: 10.1016/S0896-6273(00)80120-8

Jones, S., Livner, Å., and Bäckman, L. (2006). Patterns of prospective and retrospective memory impairment in preclinical Alzheimer's disease. Neuropsychology 20, 144-152. doi: 10.1037/0894-4105.20.2.144

Josselyn, S. A., and Nguyen, P. V. (2005). CREB, synapses and memory disorders: past progress and future challenges. Curr. Drug Targets CNS Neurol. Disord. 4, 481-497. doi: 10.2174/156800705774322058

Juhasz, G., Dunham, J. S., McKie, S., Thomas, E., Downey, D., Chase, D., et al. (2011). The CREB1-BDNF-NTRK2 pathway in depression: multiple genecognition-environment interactions. Biol. Psychiatry 69, 762-771. doi: 10.1016/ j.biopsych.2010.11.019

Kamat, R., Weinborn, M., Kellogg, E. J., Bucks, R. S., Velnoweth, A., and Woods, S. P. (2014). Construct validity of the Memory for Intentions Screening Test (MIST) in healthy older adults. Assessment 21, 742-753. doi: 10.1177/ 1073191114530774

Kandel, E. (2006). Biology of the mind. Newsweek 147, 47.

Kandel, E. R. (2012). The molecular biology of memory: cAMP, PKA, CRE, CREB-1, CREB-2, and CPEB. Mol. Brain 5:14. doi: 10.1186/1756-6606-5-14

Kandel, E. R. (2013). Principles of Neural Science. New York, NY: McGraw-Hill Medical.

Kanehisa, M. (2002). The KEGG database. Novartis Found. Symp. 247, 91-101; discussion 101-103, 119-128, 244-152. doi: 10.1002/0470857897.ch8

Kawanishi, Y., Harada, S., Tachikawa, H., Okubo, T., and Shiraishi, H. (1999). Novel variants in the promoter region of the CREB gene in schizophrenic patients. J. Hum. Genet. 44, 428-430. doi: 10.1007/s100380050196

Kennedy, K. M., Reese, E. D., Horn, M. M., Sizemore, A. N., Unni, A. K., Meerbrey, M. E., et al. (2015). BDNF val66met polymorphism affects aging of multiple types of memory. Brain Res. 1612, 104-117. doi: 10.1016/j.brainres.2014.09.044

Kliegel, M., Altgassen, M., Hering, A., and Rose, N. S. (2011). A process-model based approach to prospective memory impairment in Parkinson's disease. Neuropsychologia 49, 2166-2177. doi: 10.1016/j.neuropsychologia.2011.01.024

Lamprecht, R., Hazvi, S., and Dudai, Y. (1997). cAMP response element-binding protein in the amygdala is required for long- but not short-term conditioned taste aversion memory. J. Neurosci. 17, 8443-8450.

Lazary, J., Juhasz, G., Anderson, I. M., Jacob, C. P., Nguyen, T. T., Lesch, K. P., et al. (2011). Epistatic interaction of CREB1 and KCNJ6 on rumination and negative emotionality. Eur. Neuropsychopharmacol. 21, 63-70. doi: 10.1016/j.euroneuro. 2010.09.009

Machiela, M. J., and Chanock, S. J. (2015). LDlink: a web-based application for exploring population-specific haplotype structure and linking correlated alleles of possible functional variants. Bioinformatics 31, 3555-3557. doi: 10.1093/ bioinformatics/btv402

Mantamadiotis, T., Lemberger, T., Bleckmann, S. C., Kern, H., Kretz, O., Martin Villalba, A., et al. (2002). Disruption of CREB function in brain leads to neurodegeneration. Nat. Genet. 31, 47-54. doi: 10.1038/ng882

Mizuno, M., Yamada, K., Maekawa, N., Saito, K., Seishima, M., and Nabeshima, T. (2002). CREB phosphorylation as a molecular marker of memory processing in the hippocampus for spatial learning. Behav. Brain Res. 133, 135-141. doi: 10.1016/S0166-4328(01)00470-3 
Pittenger, C., Huang, Y. Y., Paletzki, R. F., Bourtchouladze, R., Scanlin, H., Vronskaya, S., et al. (2002). Reversible inhibition of CREB/ATF transcription factors in region CA1 of the dorsal hippocampus disrupts hippocampusdependent spatial memory. Neuron 34, 447-462. doi: 10.1016/S0896-6273(02) 00684-0

Purcell, S., Neale, B., Todd-Brown, K., Thomas, L., Ferreira, M. A., Bender, D., et al. (2007). PLINK: a tool set for whole-genome association and population-based linkage analyses. Am. J. Hum. Genet. 81, 559-575. doi: 10.1086/519795

Quinn, P. G., and Granner, D. K. (1990). Cyclic AMP-dependent protein kinase regulates transcription of the phosphoenolpyruvate carboxykinase gene but not binding of nuclear factors to the cyclic AMP regulatory element. Mol. Cell. Biol. 10, 3357-3364. doi: 10.1128/MCB.10.7.3357

R Development Core Team (2015). R: A Language and Environment for Statistical Computing [Internet]. Vienna: R Foundation for Statistical Computing.

Rankinen, T., Argyropoulos, G., Rice, T., Rao, D. C., and Bouchard, C. (2010). CREB1 is a strong genetic predictor of the variation in exercise heart rate response to regular exercise the HERITAGE family study. Circulation 3, 294-299. doi: 10.1161/CIRCGENETICS.109.925644

Raskin, S. A., Buckheit, C. A., and Waxman, A. (2012). Effect of type of cue, type of response, time delay and two different ongoing tasks on prospective memory functioning after acquired brain injury. Neuropsychol. Rehabil. 22, 40-64. doi: 10.1080/09602011.2011.632908

Sands, W. A., and Palmer, T. M. (2008). Regulating gene transcription in response to cyclic AMP elevation. Cell. Signal. 20, 460-466. doi: 10.1016/j.cellsig.2007.10. 005

Saura, C. A., and Valero, J. (2011). The role of CREB signaling in Alzheimer's disease and other cognitive disorders. Rev. Neurosci. 22, 153-169. doi: 10.1515/ RNS.2011.018

Smith, G., Della Sala, S., Logie, R. H., and Maylor, E. A. (2000). Prospective and retrospective memory in normal ageing and dementia: a questionnaire study. Memory 8, 311-321. doi: 10.1080/09658210050117735

Squire, L. R. (1992). Declarative and nondeclarative memory: multiple brain systems supporting learning and memory. J. Cogn. Neurosci. 4, 232-243. doi: 10.1162/jocn.1992.4.3.232

Sweatt, J. D. (2009). Mechanisms of Memory [Online]. Amsterdam: Elsevier Science.

Taylor, A. K., Klisak, I., Mohandas, T., Sparkes, R. S., Li, C., Gaynor, R., et al. (1990). Assignment of the human gene for CREB1 to chromosome 2q32.3-q34. Genomics 7, 416-421. doi: 10.1016/0888-7543(90)90176-U
Tomlinson, S. P., Davis, N. J., Morgan, H. M., and Bracewell, R. M. (2014). Cerebellar contributions to verbal working memory. Cerebellum 13, 354-361. doi: 10.1007/s12311-013-0542-3

Tulving, E. (1985). Elements of Episodic Memory. Oxford: Clarendon.

Viosca, J., Malleret, G., Bourtchouladze, R., Benito, E., Vronskava, S., Kandel, E. R., et al. (2009). Chronic enhancement of CREB activity in the hippocampus interferes with the retrieval of spatial information. Learn. Mem. 16, 198-209. doi: 10.1101/lm.1220309

Wallace, D. L., Han, M. H., Graham, D. L., Green, T. A., Vialou, V., Iniguez, S. D., et al. (2009). CREB regulation of nucleus accumbens excitability mediates social isolation-induced behavioral deficits. Nat. Neurosci. 12, 200-209. doi: 10.1038/nn.2257

Williams, C. M., El Mohsen, M. A., Vauzour, D., Rendeiro, C., Butler, L. T., Ellis, J. A., et al. (2008). Blueberry-induced changes in spatial working memory correlate with changes in hippocampal CREB phosphorylation and brainderived neurotrophic factor (BDNF) levels. Free Radic. Biol. Med. 45, 295-305. doi: 10.1016/j.freeradbiomed.2008.04.008

Woods, S. P., Moran, L. M., Dawson, M. S., Carey, C. L., Grant, I., and The HIV Neurobehavioral Research Center (HNRC) Group (2008). Psychometric characteristics of the memory for intentions screening test. Clin. Neuropsychol. 22, 864-878. doi: 10.1080/138540407015 95999

Woods, S. P., Twamley, E. W., Dawson, M. S., Narvaez, J. M., and Jeste, D. V. (2007). Deficits in cue detection and intention retrieval underlie prospective memory impairment in schizophrenia. Schizophr. Res. 90, 344-350. doi: 10. 1016/j.schres.2006.11.005

Conflict of Interest Statement: The authors declare that the research was conducted in the absence of any commercial or financial relationships that could be construed as a potential conflict of interest.

Copyright (c) 2017 Avgan, Sutherland, Lea, Spriggens, Haupt, Shum and Griffiths. This is an open-access article distributed under the terms of the Creative Commons Attribution License (CC BY). The use, distribution or reproduction in other forums is permitted, provided the original author(s) or licensor are credited and that the original publication in this journal is cited, in accordance with accepted academic practice. No use, distribution or reproduction is permitted which does not comply with these terms. 